\title{
Analysis of radiation-induced small Cu particle cluster formation in aqueous $\mathrm{CuCl} 2$
}

Sumedha Jayanetti

Missouri State University

Robert A. Mayanovic

Missouri State University

W A. Bassett

A J. Anderson

I M. Chou

Follow this and additional works at: https://bearworks.missouristate.edu/articles-cnas

\section{Recommended Citation}

Jayanetti, Sumedha, Robert A. Mayanovic, Alan J. Anderson, William A. Bassett, and I-Ming Chou. "Analysis of radiation-induced small $\mathrm{Cu}$ particle cluster formation in aqueous $\mathrm{CuCl} 2$." The Journal of Chemical Physics 115, no. 2 (2001): 954-962.

This article or document was made available through BearWorks, the institutional repository of Missouri State University. The work contained in it may be protected by copyright and require permission of the copyright holder for reuse or redistribution.

For more information, please contact BearWorks@library.missouristate.edu. 


\title{
Analysis of radiation-induced small $\mathrm{Cu}$ particle cluster formation in aqueous $\mathrm{CuCl}_{2}$
}

\author{
Sumedha Jayanetti ${ }^{\mathrm{a})}$ and Robert A. Mayanovic ${ }^{\mathrm{b})}$ \\ Department of Physics, Astronomy and Materials Science, Southwest Missouri State University, Springfield, \\ Missouri 65804
}

\author{
Alan J. Anderson \\ Department of Geology, St. Francis Xavier University, P. O. Box 5000, Antigonish, Nova Scotia, B2G 2W5, \\ Canada \\ William A. Bassett \\ Department of Geological Sciences, Cornell University, Ithaca, New York 14853 \\ I.-Ming Chou \\ MS 954, U.S. Geological Survey, Reston, Virginia 20192
}

(Received 8 January 2001; accepted 25 April 2001)

\begin{abstract}
Synchrotron x-ray radiation is being used extensively as a structure probe to investigate the coordination environment and thus gain insight into the ion-water and ion-ion interactions in aqueous solutions. However, under favorable conditions, there may be instances where the incident x-ray beam can induce oxidation and/or reduction in the solution, thus altering its chemistry. Successive $\mathrm{x}$-ray absorption fine structure spectra, measured in the fluorescence mode from a 55 ppm $\mathrm{Cu}$ in $\mathrm{CuCl}_{2}$ aqueous solution, show the formation of copper clusters and their growth as a function of time of irradiation. Initially, the clusters have a nearest neighbor distance of $2.48 \pm 0.02$ $\AA$ which, with increase in time of irradiation, increases to $2.55 \pm 0.01 \AA$, indicating that the clusters approach the lattice dimensions of bulk copper. Similarly, the Debye-Waller factor of the copper clusters is found to increase by $\sim 50 \%-55 \%$ over the range of time of irradiation. Analysis of spectra measured in the intermediate time period shows signal contributions from a mixture of clusters that can be represented by a mixture of a small cluster ( $5-10 \AA$ across) and bulk copper. The nearest neighbor coordination number is found to increase in a manner consistent with the decrease in the surface to volume ratio as the average cluster size approaches its bulk dimensions. The initiation of cluster growth occurs through agglomeration of copper atoms that possibly react to form dimers upon reaction. The copper ions in the solution are reduced to the metallic state by reacting with hydrated electrons produced as a result of radiolysis of water by the incident $\mathrm{X}$-ray beam. () 2001 American Institute of Physics. [DOI: 10.1063/1.1379758]
\end{abstract}

\section{INTRODUCTION}

Application of $\mathrm{x}$-ray synchrotron radiation as a structure probe in aqueous chemistry and geochemistry has become an important technique during the past decade. With the advent of third generation synchrotron radiation facilities that are capable of generating $\mathrm{x}$-ray micro beams of high brilliance, having intensities two to three orders of magnitude greater than second generation sources, there is a growing interest towards such applications among physicists, chemists, geophysicists, and geochemists. The associated $\mathrm{x}$-ray techniques have yielded new information that is of great importance in the above areas and related fields. Yet, in the case of aqueous solutions, there may be instances where the incident $\mathrm{x}$-ray beam may alter the conditions of the experiment and lead to changes in chemical properties of the sample. This typically occurs due to effects that $\mathrm{x}$-rays have on water. The extent to which these affect the chemistry of the solution is controlled

\footnotetext{
${ }^{a)}$ On leave from Department of Physics, University of Colombo, Colombo 3, Sri Lanka.

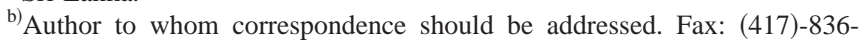
6226; electronic mail: ram051f@smsu.edu
}

especially by factors such as the time of exposure to the incident x-ray beam, the x-ray beam flux, and the chemical and physical properties of the solution itself.

A detailed discussion of the effect of radiation on aqueous solutions is beyond the scope of this paper. Jonah ${ }^{1}$ has written a concise review on progress in the field of aqueous radiation chemistry. Therein he states that intense x-rays and gamma rays cause radiolysis of water in aqueous solutions producing primarily species such as $\mathrm{H}^{+}, \mathrm{H}, \mathrm{OH}$, and $\mathrm{OH}^{-}$ and hydrated electrons, $e_{\mathrm{aq}}^{-}$. These transient species interact with themselves, water, the solute components or the free radicals that are generated by irradiation. The reaction mechanisms may be governed by the properties of the solution such as the $\mathrm{pH}$, the type of solute, its concentration and the stabilizing agents added to the solution.

Several spectrophotometric studies have been made on copper complexes in aqueous solutions undergoing radiolysis. Freiberg et al. ${ }^{2}$ reported that the reaction of aliphatic free radicals with copper cations in aqueous solutions occurs on account of pulse radiolysis. Formation of colloidal copper particles due to the reduction of $\mathrm{Cu}^{2+}$ in aqueous solutions of $\mathrm{Cu}(\mathrm{ClO})_{4}$ and $\mathrm{HCOONa}$ by pulse-radiolysis has been re- 
ported by Ershov et al. ${ }^{3}$ Henglein, ${ }^{4}$ reported on the formation of copper nanoparticles due to the radiolytic reduction of $\mathrm{Cu}^{2+}$ by $\gamma$-irradiation of aqueous solutions of $\mathrm{KCu}(\mathrm{CN})_{2}$ which contained methanol or 2-propanol as an $\mathrm{OH}$ scavenger. It is interesting to note that in the above studies, in addition to the role played by $\mathrm{H}^{+}, \mathrm{H}, \mathrm{OH}, \mathrm{OH}^{-}$, and $e_{\mathrm{aq}}^{-}$, the hydrated electron, the reduction of copper cations is achieved via the direct or indirect involvement of an organic ion or an organic-free radical generated in the solution due to irradiation.

In contrast, the present study focuses on the radiationinduced copper particle formation where there is no involvement of such organic components in the solution. The material of interest in this study, a dilute aqueous solution of $\mathrm{CuCl}_{2}$, was irradiated by intense $\mathrm{x}$-rays which were used to simultaneously monitor the structural changes by means of $\mathrm{x}$-ray absorption fine structure (XAFS) spectroscopy. Due to its ability to probe the local short-range structure, XAFS is proven to be applicable to fluids and aqueous solutions. The technique has been successfully applied to investigate the formation of small metallic particle clusters on solid substrates in a gaseous environment. Apai et al., ${ }^{5}$ have applied XAFS to investigate copper and nickel clusters formed on amorphous carbon substrates using vapor deposition. The authors reported a contraction of near-neighbor distance and an increase in binding energy for smaller copper and nickel clusters. Montano et al., ${ }^{6}$ have observed similar nearneighbor distance contractions in copper clusters grown on carbon substrates using the gas aggregation technique. Marcus et al., ${ }^{7}$ and Balerna et al., ${ }^{8}$ applied XAFS to characterize small clusters of gold on Mylar substrates. However, to our knowledge, this is the first attempt at using XAFS to investigate such behavior in an aqueous environment where the incident $\mathrm{x}$-ray beam induces the formation of metallic clusters. The XAFS technique, being element specific, allows for the selection of individual element absorption spectra within materials of complex composition. A simple system was chosen for this study in order to minimize the possible complications that might arise due to radiation-induced changes in the chemistry of a more complex system.

\section{EXPERIMENT}

The sample irradiation and the $\mathrm{Cu} K$-edge XAFS spectra were measured from the samples at the undulator PNC-CAT ID20 beam line at the Advanced Photon Source, Argonne National Lab. The synchrotron was operated at $7.0 \mathrm{GeV}$ and $100 \mathrm{~mA}$ maximum fill current. Energy calibration was accomplished using a copper foil. The crystals of the monochromator were detuned by $20-30 \%$ in order to reduce the harmonic content in the incident $\mathrm{x}$-ray beam. KirkpatrickBaez mirrors were used to focus the incident $\mathrm{x}$-ray beam, giving a roughly circular spot measuring $\sim 20 \mu \mathrm{m}$ across at the focal point. The sample used was a $55 \mathrm{ppm} \mathrm{Cu}$ in aqueous solution of $\mathrm{CuCl}_{2}$ enclosed in a hydrothermal diamond anvil cell. Since trace quantities of copper chloride were used, it was imperative that the solution was placed in a cell that would not add contaminants to the sample through corrosion or irradiation. ${ }^{9}$ The solution was sealed between the

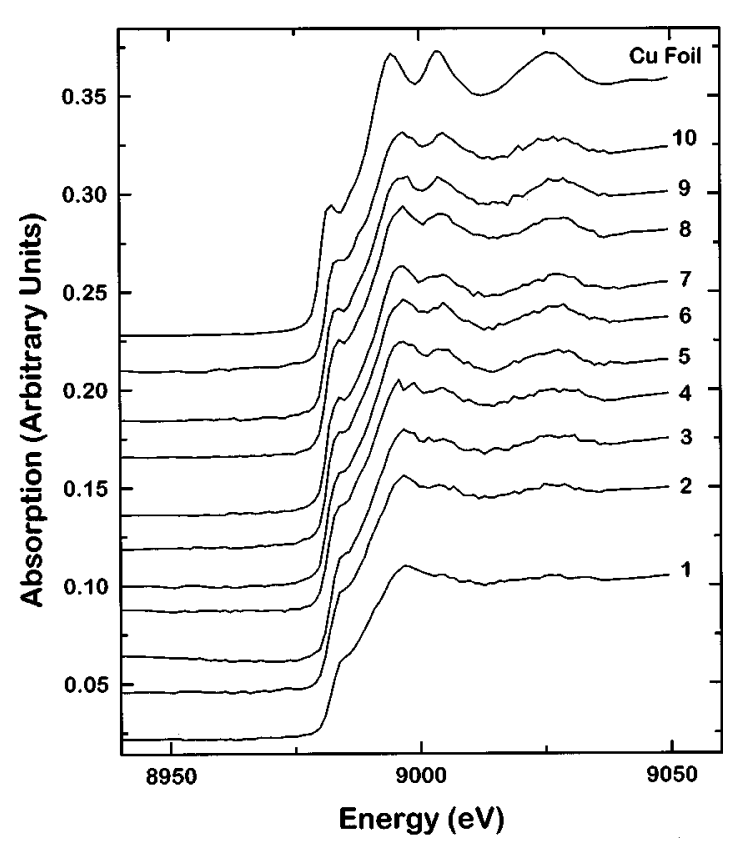

FIG. 1. The near edge (XANES) range of the ten successive $\mathrm{Cu} K$-edge XAFS spectra beginning from spectrum number 1 (the first collected spectrum). The XANES spectrum of $\mathrm{Cu}$ foil (reduced by a factor $1 / 20$ ) is shown for comparison.

upper and lower diamond anvils in the sample chamber consisting of a $300 \mu \mathrm{m}$ diameter cup-shaped cavity in the center of the upper diamond anvil face and a $300 \mu \mathrm{m}$ diameter hole in a $50 \mu \mathrm{m}$ thick Re gasket. The solution was then irradiated by an x-ray beam that travelled through the upper diamond in a direction parallel to the anvil face. The XAFS spectra were collected in the fluorescence mode, in the standard $90^{\circ}$ orientation to the incident $\mathrm{x}$-ray beam, using a thirteenelement Ge detector. The undulator gap voltage was maintained at $55 \mathrm{eV}$ throughout the collection of data, giving a flux of approximately $5 \times 10^{11}$ photons/s, and ten successive XAFS spectra were collected within a duration of approximately $6 \mathrm{~h}$.

\section{DATA ANALYSIS AND RESULTS}

Shown in Fig. 1 is the near edge (XANES) range of the successive $\mathrm{Cu} K$-edge XAFS spectra starting from spectrum number 1 (the first measured spectrum). From the figure, the evolving multiple scattering features that correspond to the changes in the evolving growth and coordination environment around the $\mathrm{Cu}$ absorber can be seen clearly. A similar increase in amplitude can be seen in the XAFS range of alternate spectra named A, B, C, D, and E (spectra 1, 3, 5, 7, and 9, respectively) with others removed for clarity in Fig. 2. In both Figs. 1 and 2, it is evident that the fine structure in the spectra evolves with time to reflect more of the features of the spectrum measured from the $\mathrm{Cu}$ foil. For all spectra, the maximum point of the derivative spectrum was chosen as the $\mathrm{Cu} \mathrm{K}$-edge threshold energy. These showed a gradual shift towards higher values when going from $\mathrm{E}$ to A with spectrum A showing the maximum shift of $0.96 \mathrm{eV}$ with respect to E (see Table I). Prior to data analysis, the XAFS range of each spectrum above $9040 \mathrm{eV}$ was smoothed by 2 -point adjacent averaging in order to minimize the noise. 


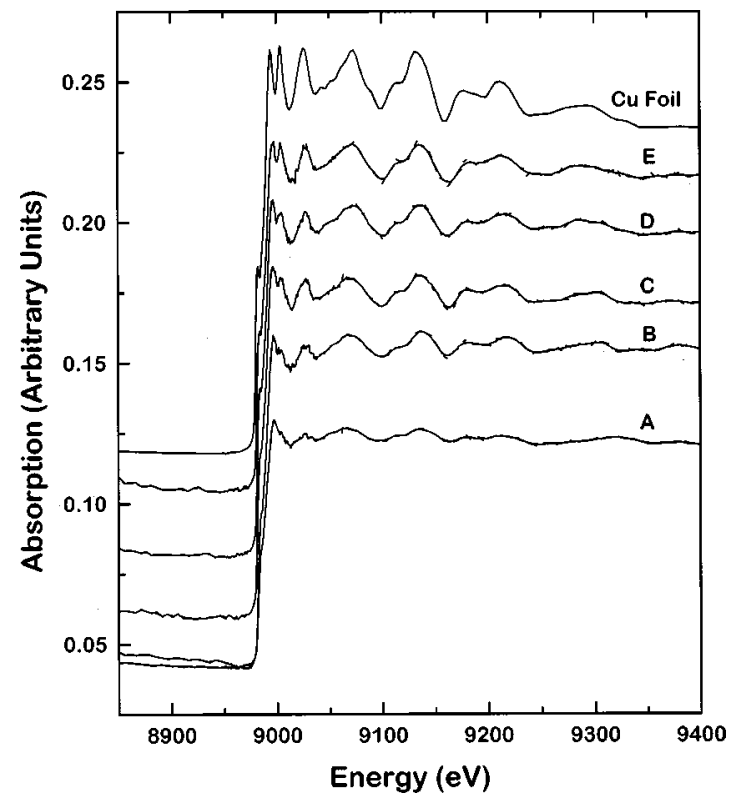

FIG. 2. XAFS range of alternate spectra named A, B, C, D, and E (spectra $1,3,5,7$, and 9, respectively, in Fig. 1) with others removed for clarity. XAFS range of each spectrum above $9040 \mathrm{eV}$ was smoothed by two-point adjacent averaging. The solid lines represent smoothed data with the raw XAFS data shown as dashed lines. The XAFS spectra of $\mathrm{Cu}$ foil (reduced by a factor $1 / 20$ ) is shown for comparison.

The solid lines in Fig. 2, where the raw data are shown as dashed lines, represent the smoothed XAFS data and show that smoothing does not cause any significant distortion in the signal. The data were then normalized with respect to the incident beam and the XAFS oscillations, $\chi(k)$, were extracted using the automated background subtraction program AUTOBK. ${ }^{10}$ The data analysis was done using FEFFIT (version 2.54) analysis program ${ }^{11,12}$ that employs a nonlinear, least square fit to the theoretical standards calculated using FEFF8 theoretical code ${ }^{13}$ Fitting parameters included the coordination number $\left(N_{i}\right)$, the radial distance $\left(R_{i}\right)$, the XAFS Debye-Waller factor $\left(\sigma_{i}^{2}\right)$, where index $i$ represents the $i$ th coordination shell, and $\Delta E_{0}$ which compensates for any discrepancy between the experimental edge energy and its theoretical estimate. The quality of a fit is indicated by the $\mathfrak{R}$-factor, which is normally less than 0.05 for a good fit.

Shown in Fig. 3 are the Fourier transforms of $\chi(k)$ data of spectra A, B, C, D, \& E, respectively, in comparison with

TABLE I. The $\mathrm{Cu} K$-edge threshold energy, as determined from the maximum point of the derivative, the energy shift relative to $K$-edge threshold energy of spectrum E, and the step height of XAFS spectra collected at regular intervals from a $55 \mathrm{ppm} \mathrm{Cu}$ dissolved in $\mathrm{CuCl}_{2}$ aqueous solution undergoing beam-induced reduction and copper cluster formation.

\begin{tabular}{cccc}
\hline $\begin{array}{c}\text { Spectrum } \\
\text { identification }\end{array}$ & $\begin{array}{c}K \text {-edge threshold } \\
\text { energy (eV) }\end{array}$ & $\begin{array}{c}\text { Energy }^{\mathrm{a}} \\
\text { shift }(\mathrm{eV})\end{array}$ & $\begin{array}{c}\text { Step } \\
\text { height }\end{array}$ \\
\hline A & 8982.23 & 0.96 & 0.083 \\
B & 8982.00 & 0.73 & 0.114 \\
C & 8981.40 & 0.13 & 0.118 \\
D & 8981.33 & 0.06 & 0.121 \\
E & 8981.27 & 0.00 & 0.119 \\
\hline \hline
\end{tabular}

${ }^{a}$ Energy shift was evaluated with respect to $K$-edge threshold energy of spectrum E.

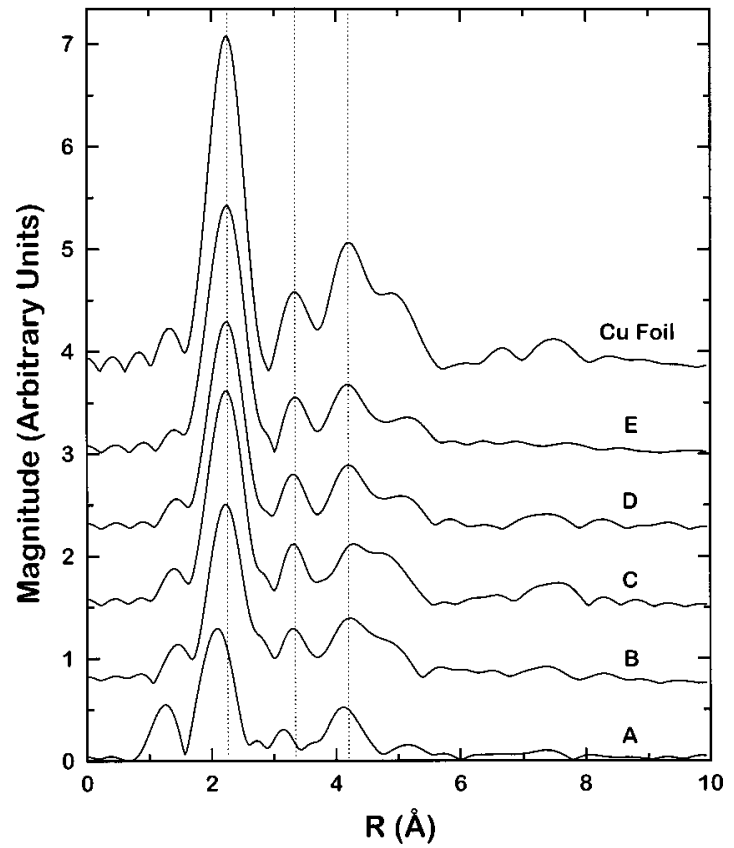

FIG. 3. The magnitude of the Fourier transforms of $k^{2} \cdot \chi(k)$ data obtained from the XAFS spectra A, B, C, D, \& E and that of Cu foil. Note the shifts of the peaks towards lower $r$ values in spectrum A compared to the other spectra.

the Fourier transform of the $\mathrm{Cu}$ foil $\chi(k)$ data. These data have been transformed to $r$-space by using a Hanning window on $\chi(k)$ data with $d k=1.0 \AA^{-1}$, in the $k$ range $2.5-$ $10.5 \AA^{-1}$ and $k^{2}$ weighting. It can be seen from the figure that an increase in the amplitude occurs with time of irradiation, especially in the first shell Fourier transform. It can also be seen that the peaks in spectrum A show a noticeable shift towards lower $r$ values. The principal features of the Fourier transforms of spectra B to E shown in Fig. 3 resemble those of data measured from bulk copper, at least up to the third peak. Spectra D and E show features beyond the third peak, which by appearance are most likely attributed to overlapped fourth and fifth peaks. Similar features appear in spectra B and $\mathrm{C}$, but are almost solely due to fourth shell contributions.

The common feature in the Fourier transform data is that each has significant peaks, at least up to the third one. Based on this, and the fact that each of the peaks is dominated by signal originating from photoelectron single scattering to individual coordination shells relative to the ionized, central atom, it was decided that fitting of the data was to be made to include the range up to the third shell peak. Thus, the major objective of the study was to compare the fitting results of spectrum A with the rest of the spectra measured from the solution sample. For this reason, a four-shell face-centered cubic (fcc) model was used as the standard for fitting, taking all of the significant scattering paths, including the multiple scattering paths, into account. Because this model suffices to adequately fit spectra collected from bulk copper, we will refer to it as the "bulk model." Fits were attempted alternately on Fourier transforms in $r$-space and on the backtransformed (filtered) data $[\tilde{\chi}(k)]$ in $k$-space by filtering the Fourier transform data in the $r$ range of 1.5-5.8 $\AA$ with $k^{2}$ weighting. Parameters $\left(\Delta E_{0}=5.0 \mathrm{eV}, S_{0}^{2}=0.9\right.$, and the 
TABLE II. The theoretically determined values of near neighbors up to third-shell for clusters having atoms ranging from 6 to 55 in the fcc configuration in comparison with the corresponding values for bulk copper.

\begin{tabular}{lccc}
\hline \hline \multirow{2}{*}{$\begin{array}{l}\text { No. of atoms } \\
\text { in the cluster }\end{array}$} & \multicolumn{3}{c}{ Coordination number } \\
\cline { 2 - 4 } & 1st shell & 2nd shell & 3rd shell \\
\hline 6 & 4 & 1 & 0 \\
13 & 5.54 & 1.89 & 3.69 \\
19 & 6.32 & 1.89 & 5.05 \\
43 & 7.26 & 3.07 & 8.93 \\
55 & 7.85 & 3.27 & 9.60 \\
Bulk copper & 12 & 6 & 24 \\
\hline
\end{tabular}

Debye-Waller factors reported in Table IV) obtained by the fitting of $\mathrm{Cu}$ foil data were found to be in good agreement with the previously reported values. ${ }^{13,14}$ When fits were attempted with the bulk model, only spectra D and E could be fit using this model alone. Fitting was also made in the filtered $k$ space using a shorter filtering range in $r$ space $(1.5-$ $5.3 \AA$ ) in order to determine what effect, if any, the overlapped fifth shell contribution to the spectra had on the fit parameters. It yielded similar results with negligible changes in the third shell structural parameters that were well within error bars of respective results. The Debye-Waller factor corresponding to the second shell was constrained at 0.01 $\left(\AA^{2}\right)$ in order to avoid fluctuations in fits giving rise to non-

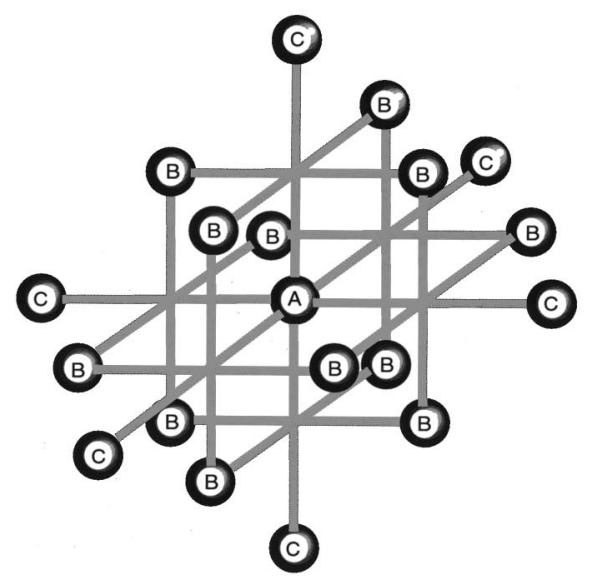

(iii)
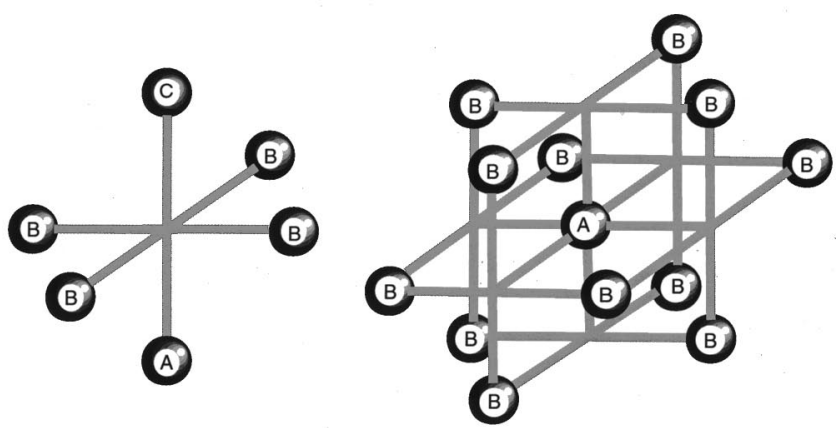

(i)

FIG. 4. An illustration of (i) 6-, (ii) 13-, and (iii) 19-atom fcc clusters used for modeling XAFS standards. For the sake of clarity 43- and 55-atom clusters are not included in the figure. The straight lines in the diagram represent the fcc lattice rather than the interatomic bonds.

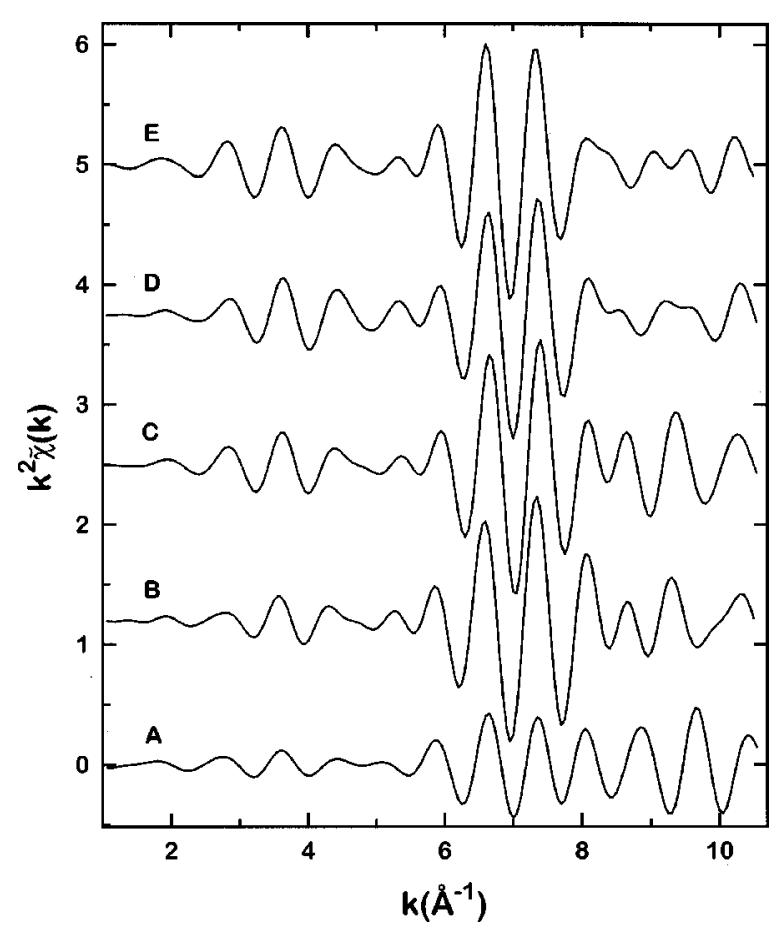

FIG. 5. Filtered data, $k^{2} \cdot \tilde{\chi}(k)$, in the higher shell range (3.0-6.0 $⿱$ ) of spectra shown in Fig. 3.

physical results. $\Delta E_{0}$ was fixed at $4.9 \mathrm{eV}$ during fitting and was found to be in good agreement with that used to fit the $\mathrm{Cu}$ foil data.

However, fitting spectra $\mathrm{A}, \mathrm{B}$, and $\mathrm{C}$ required modifications in the standard model. Attempts were made to fit with a mixture of a small copper cluster and bulk copper models. A number of clusters, ranging in size from dimers, trimers and larger, having either the fcc configuration or the body centered cubic (bcc) configuration, were considered. However, as indicated by the fitting of the spectra, fcc clusters were found to be most suitable for fitting. Table II shows the theoretically determined values of near neighbors up to third shell for clusters having atoms ranging from 6 to 55 in the fcc configuration. Shown in Fig. 4 are some of the clusters, with the larger clusters not included for the sake of clarity. Since any combination can safely be assumed to exist in the solution during the initial phase, theoretical XAFS curves were calculated for each of the clusters listed in Table II using FEFF8 code and tried as part of a standard model for fitting. The exact nature of the distribution of copper clusters at any time is governed by the reaction kinetics of the aqueous solution undergoing X-ray-beam-induced radiolysis. During calculations, the near-neighbor distances of the clusters were set at or slightly lower than bulk-copper values. However, without applying any constraints, these distances were allowed to vary during fitting. Instead of using a wide mixture of clusters, care was taken to simplify the standard model by using just one cluster along with the bulk copper model, in order to minimize the total number of fitting parameters. In this way, we were able to obtain the most relevant and direct structure information pertaining to the agglomeration of copper clusters with irradiation time.

It is apparent from Table II that any one of the 13 to 55 


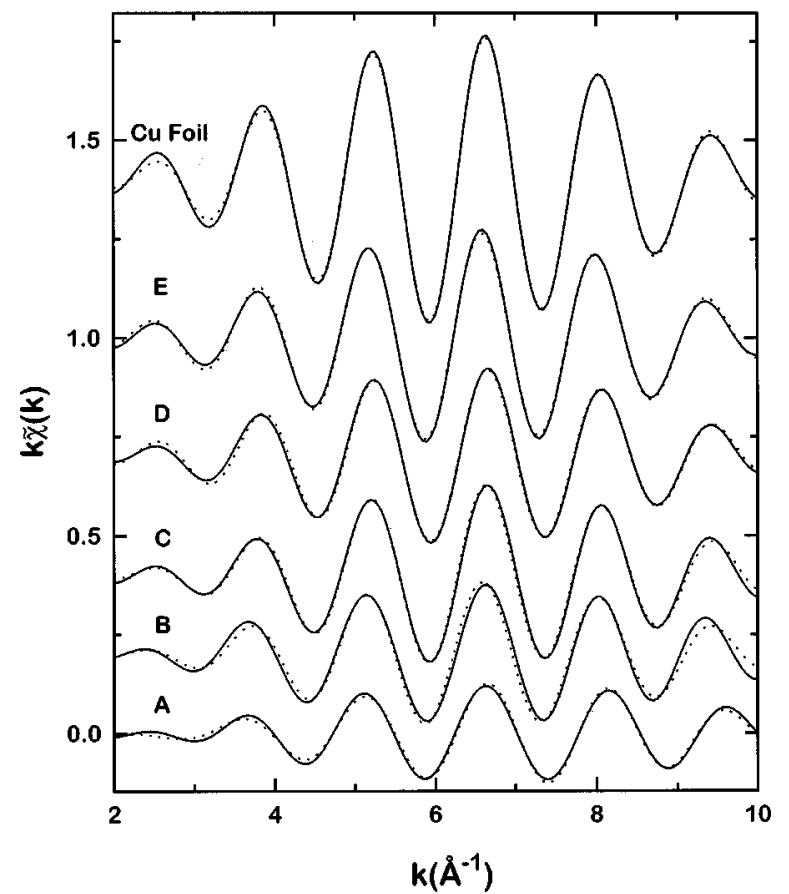

FIG. 6. Single shell fits (dashed lines) obtained for $k \cdot \widetilde{\chi}(k)$ data (solid lines) obtained by filtering the first Fourier transform peak (1.5-3.0 $⿱$ A) of spectra $\mathrm{A}-\mathrm{E}$ and the spectrum of $\mathrm{Cu}$ foil.

atom fcc clusters can have a contribution to the third shell portion of the XAFS signal. Filtered $\tilde{\chi}(k)$ data in the higher shell range (3.0-6.0 $\AA$ ) of the spectra show (Fig. 5) that the cluster contribution is significant at lower $k$ values. Thus, fits were attempted on the first shell of spectra A to $\mathrm{C}$, by filtering the Fourier transformed data in the r-range 1.5-3.0 $\AA$, with $k^{1}$ weighting, in order to enhance the details in the lower $k$ region. Shown in Fig. 6 are the single, first-shell peak fits made on the filtered $k \widetilde{\chi}(k)$ data, in $k$ space. The results from these fittings are listed in Table III. Fitting of spectrum A showed that there was essentially no bulk copper contribution towards the spectrum. Furthermore, it was observed that either one of a cluster of 19 or 43 atoms, respectively, would yield similar results (with a slightly larger $\mathfrak{R}$ factor for 43-atom cluster than for 19-atom cluster) in the fitting. The fitting parameter $\Delta E_{0}$ was found to show a preference towards negative values and was fixed at $-4.0 \mathrm{eV}$. As
Table III shows that the first-shell Debye-Waller factor $\left(\sigma^{2}\right)$, found from fitting of spectrum A, was significantly lower compared to the values obtained from fits of spectra D and $\mathrm{E}$.

For spectrum A, fitting the Fourier transformed XAFS data in the extended $r$-range of 1.5-4.7 $\AA$ (range that includes all three Fourier transform peaks in spectrum A) was favored by an fcc cluster of 19 atoms and yielded structural parameters that were consistent with the first shell parameters. The Fourier transforms were made using $k^{2}$ weighting of the $\chi(k)$ data. First shell structure parameters were maintained at the values obtained from single shell fits, in order to reduce the number of fitting variables. Due to the relatively smaller intensity of the second peak in the Fourier transformed spectrum, allowing for the variation of second-shell fitting parameters led to nonphysical results. This was circumvented by coupling the second-shell coordination number to that of the first shell according to the theoretically determined coordination numbers of the 19-atom cluster. Also, fourth-shell scattering paths were considered in order to account for a possible, relatively small overlap of the third-shell peak with the fourth-shell peak. The fourth-shell type of feature (seen most easily in the Fourier transform data shown in Fig. 3) was seen to be dominated by the triple scattering of the photoelectron from the first near-neighbor atoms rather than by the single scattering from fourth-shell atoms. This is completely consistent with our finding that the 19-atom cluster is the most suitable fitting model for Spectrum A.

Fitting made of the Fourier transform data (excluding spectra $\mathrm{B}$ and $\mathrm{C}$ ) over the multiple peaks in the $r$ space (spectrum A: $1.5-4.7 \AA$, spectra D \& E: $1.5-5.8 \AA$ ), and of the corresponding filtered $\tilde{\chi}(k)$ data over the extended $k$ range $\left(2.5-10.15 \AA^{-1}\right)$ are shown in Figs. $7($ a) and $7(b)$, respectively. The corresponding structure results from these fittings are listed in Table IV. Spectra B and C were fit with a mixture of a 19-atom cluster and bulk copper models with the fitting limited in the range of the first peak only, in order to avoid the inaccuracies that contribute to the fitting parameters due to correlations of a larger number of fit variables. However, while the first peak was fit successfully, attempts at fitting the wider range of $r$-space over the multiple peaks showed that a two-model standard was insufficient. Fitting

TABLE III. First-coordination shell structure parameters of copper clusters at various stages of agglomeration, as determined from fitting of Cu $K$-edge XAFS spectra collected at regular intervals from a $55 \mathrm{ppm} \mathrm{Cu}$ dissolved in $\mathrm{CuCl}_{2}$ aqueous solution. The same structure parameter results from fitting of the $\mathrm{Cu}$ foil spectrum are shown for comparison. $N_{1}$ is the coordination number, $R_{1}$ the relative $\mathrm{Cu}$-Cu near-neighbor distance, and $\sigma_{1}^{2}$ is the Debye-Waller harmonic disorder factor. $N_{\text {total }}$ was obtained by summing the cluster and bulk model coordination number contributions. Dashes indicate that no contribution was made from this model in the fitting. The near neighbor coordination numbers, $N_{1 \text { (Cluster) }}$ and $N_{1 \text { (Bulk) }}$, reflect the relative contribution from the 19-atom cluster and bulk copper models, respectively, towards spectra B and C, rather than the actual bonds associated with the cluster and bulk copper.

\begin{tabular}{|c|c|c|c|c|c|c|c|c|c|c|}
\hline Spectrum & $N_{\text {total }}$ & $\begin{array}{c}\% \\
\text { (cluster) }\end{array}$ & $N_{1 \text { (Cluster) }}$ & $R_{1 \text { (Cluster) }}$ & $\sigma_{1 \text { (Cluster) }}^{2}$ & $\begin{array}{c}\% \\
\text { (bulk) }\end{array}$ & $N_{1 \text { (Bulk) }}$ & $R_{1 \text { (Bulk) }}$ & $\sigma_{1(\mathrm{Bulk})}^{2}$ & $\begin{array}{c}\mathfrak{R} \\
\text { factor }\end{array}$ \\
\hline A & $2.4 \pm 1.5$ & 100.0 & $2.4 \pm 1.5$ & $2.48 \pm 0.01$ & $0.0036 \pm 0.0021$ & $\cdots$ & $\cdots$ & $\cdots$ & $\cdots$ & 0.025 \\
\hline B & $4.9 \pm 1.5$ & 24.0 & $1.2 \pm 0.8$ & $2.48 \pm 0.02$ & $0.0035^{\mathrm{a}}$ & 76.0 & $3.7 \pm 1.3$ & $2.55 \pm 0.01$ & $0.0078^{\mathrm{a}}$ & 0.039 \\
\hline $\mathrm{C}$ & $6.5 \pm 1.1$ & 12.0 & $0.8 \pm 0.8$ & $2.48 \pm 0.02$ & $0.0035^{\mathrm{a}}$ & 88.0 & $5.7 \pm 0.8$ & $2.55 \pm 0.01$ & $0.0078^{\mathrm{a}}$ & 0.010 \\
\hline $\mathrm{D}$ & $6.9 \pm 0.7$ & $\cdots$ & $\cdots$ & $\cdots$ & $\cdots$ & 100.0 & $6.9 \pm 0.7$ & $2.55 \pm 0.01$ & $0.0077 \pm 0.0008$ & 0.005 \\
\hline $\mathrm{E}$ & $8.4 \pm 0.9$ & $\cdots$ & $\cdots$ & $\cdots$ & $\cdots$ & 100.0 & $8.4 \pm 0.9$ & $2.55 \pm 0.01$ & $0.0079 \pm 0.0009$ & 0.004 \\
\hline $\mathrm{Cu}$ foil & $11.8 \pm 1.0$ & $\cdots$ & $\ldots$ & $\cdots$ & $\cdots$ & 100.0 & $11.8 \pm 1.0$ & $2.55 \pm 0.01$ & $0.0084 \pm 0.0002$ & 0.003 \\
\hline
\end{tabular}

${ }^{a}$ These values were fixed during fitting. 

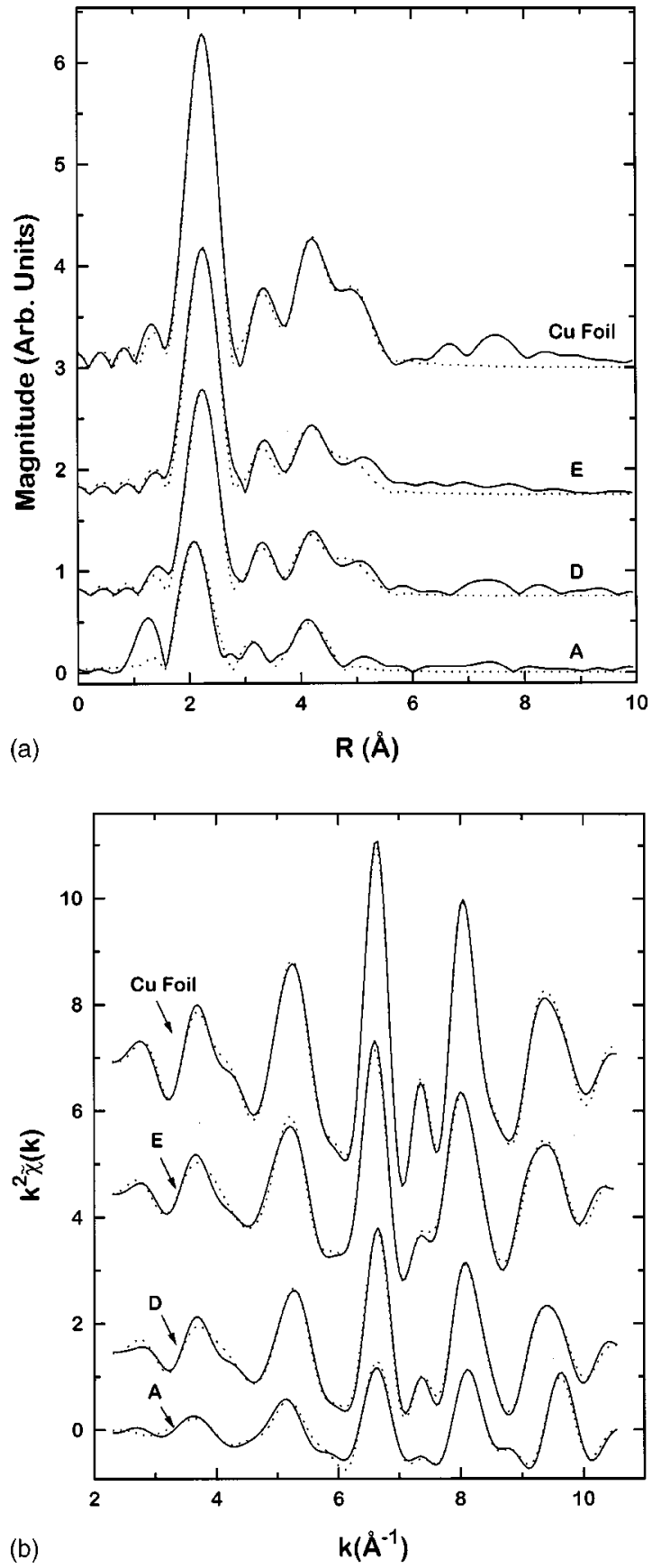

FIG. 7. (a) Multiple shell fits (dashed lines) in the $r$ space for data (solid lines) in the range 1.5-4.7 $\AA$ for spectrum $\mathrm{A}$ and in the range 1.5-5.8 $\AA$ for spectra D \& E. (b) Multiple shell fits (dashed lines) obtained for $k^{2} \cdot \tilde{\chi}(k)$ data (solid lines) obtained by filtering the Fourier transform peaks in the range $1.5-4.7$ for spectrum $\mathrm{A}$ and in the range $1.5-5.8 \AA$ for spectra $\mathrm{D} \& \mathrm{E}$.

with a multiple-cluster model over the extended $r$ range of spectra $\mathrm{B}$ and $\mathrm{C}$ proved to be too unwieldy and was, thus, abandoned. The fact that the first-near-neighbor coordination number $N_{1 \text { (Cluster) }}$ of the 19-atom cluster is decreasing, while the same number for the bulk copper model $N_{1 \text { (Bulk) }}$ is increasing for spectra $\mathrm{B}$ and $\mathrm{C}$ in Table III, reflects that the clusters in the solution are growing larger in size with elapsed time. Fits attempted for spectra D and E with a 19atom cluster and bulk copper models showed that there was essentially no contribution from the 19-atom cluster model. Furthermore, the fact that these spectra were fit satisfactorily justifies using the 4-shell bulk copper model. These results indicate that the size of the clusters in the solution increases steadily with elapsed time, to the point that the average size of the clusters after six hours of $x$-ray beam exposure, under conditions of this experiment, is at least or greater than 55 atoms.

\section{DISCUSSION}

\section{A. XAFS fitting parameters and cluster size estimates}

The coordination number results obtained from fitting are consistent with the fact that the surface to volume ratio of the clusters decreases with further agglomeration of copper particles, eventually leading to bulk-like clusters. However, comparison of the coordination number fitting results for spectrum A with calculated values for a 19-atom cluster (Table II) shows these to be significantly reduced. Our results from fitting spectrum A show a first-shell coordination number that is fairly close to the calculated value for a six-atom cluster, whereas the third shell results are consistent with that of a 13/19-atom cluster. Conversely, as stated above, fitting of spectrum A showed that a 19-atom model cluster was most appropriate. It is likely that a single-cluster fitting model used in this case acts as an average representation of a mixture of clusters existing in the sample with a 19-atom cluster being predominant. Reductions in experimental coordination numbers to calculated values were observed by Montano et al., ${ }^{6}$ for copper clusters grown on carbon substrates and were attributed to vacancies. There is a strong possibility that vacancies play a similar role in decreasing the coordination number in our case. Existence of vacancies further supports our observation of decreased Debye-Waller factor values relative to those for bulk copper, that were obtained based on analysis of spectrum A as discussed in the following.

Fitting results show that the Debye-Waller factors of the 19-atom cluster are $\sim 50-55 \%$ less than those of the bulklike clusters. This is consistent with the Debye-Waller factors obtained by Marcus et al. ${ }^{7}$ for 55 -atom gold clusters which show a thermal vibration factor of $43 \%$ less than that of bulk. Our Debye-Waller factor results are also consistent with those of Apai et al., ${ }^{5}$ who observe no disorder effects with decreasing size of copper and nickel clusters, but contradict the results obtained by Balerna et al. ${ }^{8}$ for gold clusters. Absence of disorder is further supported by the fact that attempts to include the $\mathrm{C}_{3}$ anharmonic parameter in the cumulant expansion to the fitting of spectrum $\mathrm{A}$ in the vicinity of the third-shell peak showed its value to be negligible to nonexistent.

Shifts in $K$-edge threshold energy similar to that observed in our work have previously been reported by Apai et al. ${ }^{5}$ for copper and nickel clusters grown on graphite substrates. The authors attribute these to the associated shifts in atomic core level binding energies relative to the Fermi level of the bulk material. High surface to volume ratio of the smaller clusters results in an average reduction of the number of nearest neighbors and a dominance of surface atoms, which is thought to lead to a reduction of repulsive interactions between the atoms involved. This in turn leads to an 
TABLE IV. Second- and third-coordination shell structure parameters of copper clusters at the initial (A) and later (D and E) stages of agglomeration, obtained from fitting of $\mathrm{Cu} K$ edge XAFS spectra collected from a 55 ppm $\mathrm{Cu}$ dissolved in $\mathrm{CuCl}_{2}$ aqueous solution. The same structure parameter results from fitting of the $\mathrm{Cu}$ foil spectrum are shown for comparison. $N_{2}$ and $N_{3}$ are the second- and third-coordination shell numbers, $R_{2}$ and $R_{3}$ the second- and third-neighbor distances, and $\sigma_{2}^{2}$ and $\sigma_{3}^{2}$ are the second- and third-shell Debye-Waller factors, respectively.

\begin{tabular}{lccccccc}
\hline \hline Spectrum & \multicolumn{1}{c}{$N_{2}$} & \multicolumn{1}{c}{$N_{3}$} & \multicolumn{1}{c}{$R_{2}$} & \multicolumn{1}{c}{$R_{3}$} & \multicolumn{1}{c}{$\sigma_{2}^{2}$} & $\sigma_{3}^{2}$ & $\begin{array}{c}\Re \\
\text { factor }\end{array}$ \\
\hline A (cluster) & $1.07^{\mathrm{a}}$ & $4.5 \pm 1.0$ & $3.42 \pm 0.01$ & $4.39 \pm 0.03$ & $0.0041 \pm 0.0026$ & $0.0045 \pm 0.0020$ & 0.026 \\
D (bulk) & $4.0 \pm 0.7$ & $13.3 \pm 1.6$ & $3.59 \pm 0.01$ & $4.44 \pm 0.01$ & $0.01^{\mathrm{a}}$ & $0.0120 \pm 0.0040$ & 0.014 \\
E (bulk) & $4.3 \pm 1.1$ & $13.9 \pm 2.1$ & $3.57 \pm 0.01$ & $4.44 \pm 0.01$ & $0.01^{\mathrm{a}}$ & $0.0120 \pm 0.0030$ & 0.016 \\
Cu foil & $6.3 \pm 0.87$ & $19.7 \pm 5.1$ & $3.59 \pm 0.01$ & $4.45 \pm 0.01$ & $0.01^{\mathrm{a}}$ & $0.0097 \pm 0.006$ & 0.005 \\
\hline \hline
\end{tabular}

${ }^{a}$ These values were fixed during fitting.

increase in the charge transfer process and a change in the electronic structure of the clusters, consequently strengthening the bonds between the atoms. This can be explained by characterizing the atom-atom bonding in the clusters as being somewhat more covalent-like in comparison to the (metallic) bond exhibited in the bulk metal. This has been confirmed from measurements of electronic structure of copper clusters using photoelectron spectroscopy. ${ }^{15}$ Theoretical evidence supports the higher atomic-binding energies associated with the smaller cluster sizes due to their change in electronic structure. ${ }^{16-18}$ Thus, a lower Debye-Waller factor in the earlier scans can be explained as a consequence of the stronger bonding between atoms of smaller clusters. The existence of vacancies in the clusters causes further increase in the effective surface to volume ratio leading to even less repulsive interactions between atoms and is consistent with the observed Debye-Waller factor of the representative 19atom cluster in our study. The variation in the fitting parameter $\Delta E_{0}$ from 4.9 to $-4.0 \mathrm{eV}$ is most likely associated with the change in electronic structure and hence a shift in the core electron binding energy of the smaller clusters compared to that of the bulk-like clusters. The lattice contraction effect is likewise consistent with the atom-atom bonding strength increase during the early phase of cluster agglomeration.

In addition to the changes in the electronic properties, the mechanical properties of the copper clusters are similarly altered in relation to those of bulk copper. Marcus et al. ${ }^{7}$ attributed the low Debye-Waller factor results for the 55atom gold cluster to enhanced surface pressure, which is thought to lead to a volume contraction and thus an increase in the elastic moduli. When compared to bulk copper, the volume contraction of the 19-atom cluster of our study is $\sim 8.1 \%$. Using the isothermal bulk modulus data for bulk copper, ${ }^{19}$ the volume-dependent relationship for the bulk modulus developed by Anderson et al. ${ }^{20}$ and a similar approach as employed by Marcus et al., we find an increase of $\sim 61 \%$ for the bulk modulus of the 19-atom cluster of our study compared to that of bulk copper. This is in good agreement with a $63 \%$ increase of the bulk modulus of the 55atom gold cluster relative to the bulk value found by Marcus et al. Thus, the low Debye-Waller factor values can be thought of as being directly indicative of the stiffer nature of interatomic bonds of the cluster as compared to those of bulk copper.
The cluster size estimates made using the observed bond distances yield an approximate size of $7 \AA$ for the 19 -atom fcc cluster. This result is in good agreement with those obtained by Montano et al. ${ }^{6}$ where they observe a cluster size of approximately $6 \AA$ corresponding to a first near-neighbor bond distance of $2.48 \AA$. However, our results show a discrepancy with those of Apai et al.,${ }^{5}$ who observed a cluster of approximately $15 \AA$ having a similar near-neighbor distance. Results obtained from fitting spectra B and C, using a mixture of bulk copper and 19-atom cluster models as standards, can also be used to interpret an intermediate stage of cluster growth between the 19-atom cluster and the bulk-like clusters. This suggests the existence of a distribution of clusters of increasing mean size, leading up to bulk-like clusters with successive collection of the XAFS spectra. Thus, the spectra provide information on particles in a range of sizes from $\sim 5$ to $\sim 10 \AA$ beyond which the clusters are thought to show bulk like characteristics. Montano et al. ${ }^{6}$ have observed the presence of dimers in the range of different-sized clusters grown using the gas aggregation technique. However, the results from the analysis of spectra A-E do not show direct evidence for the existence of dimers, despite the fact that dimers were very likely part of the overall statistical distribution of copper clusters present in the solution at the earliest phase of our experiment.

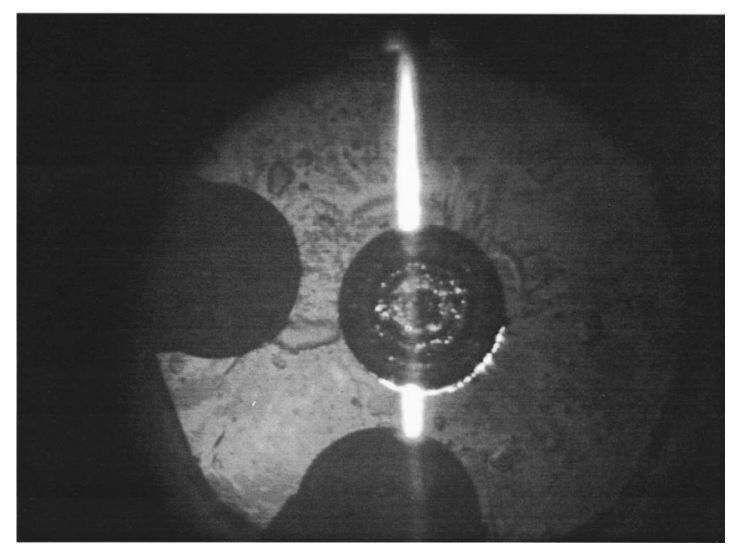

FIG. 8. Image of the visible fluorescence created by the inelastic scattering of incident $\mathrm{x}$ rays in the vicinity of the sample chamber defined by the cavity (short bright streak) and incident-groove (long bright streak) in the illuminated (top) diamond, the intervening rhenium gasket and second (bottom) diamond anvil. 


\section{B. Reduction by radiolysis}

It is interesting to investigate the role played by the incident beam during the reaction. As mentioned above, $\mathrm{H}_{2} \mathrm{O}$ during radiolysis can be activated into species such as $\mathrm{H}^{+}$, $\mathrm{H}, \mathrm{OH}, \mathrm{OH}^{-}$, and a hydrated electron, $e_{\mathrm{aq}}^{-}$thus inducing both reduction and/or oxidation in the solution depending on its properties. Boyle et al. ${ }^{21}$ have estimated that the photon energy for the formation of $e_{\mathrm{aq}}^{-}$to be $\geqslant 6.5 \mathrm{eV}$. Thus, it is more likely that the secondary radiation resulting from scattering of the incident beam by the grooved-diamond anvil led to the formation of $e_{\mathrm{aq}}^{-}$. Figure 8 illustrates the extent of this scattering by showing the fluorescence in the visible range caused by the incident $\mathrm{x}$-ray beam in the sample volume portion of the cell. The hydrated electron $e_{\mathrm{aq}}^{-}$reacts with and, thereby, causes reduction of a $\mathrm{Cu}^{2+}$ ion to its metallic state through a two-step process, i.e.,

$$
e_{\mathrm{aq}}^{-}+\mathrm{Cu}^{2+} \rightarrow \mathrm{Cu}^{+}
$$

and

$$
e_{\mathrm{aq}}^{-}+\mathrm{Cu}^{+} \rightarrow \mathrm{Cu}^{0}
$$

Although the exact kinetics are unknown, the initiation of cluster growth most likely occurs through agglomeration of copper atoms that react to form dimers upon reduction. It may be possible that, in a well-controlled experiment, using variable and less intense flux of incident beam, more understanding can be gained about the initial phase of the agglomeration including on the existence of dimers. The present study does not show evidence of $\mathrm{Cu}^{2+}$ in association with chlorine ions or oxygens in the solution.

The present work cannot provide information about the general conditions that are important for beam-induced oxidation/reduction effects and, in this context, a more detailed study is required as the conditions may vary from system to system. We have made a test of dependence of the rate of copper ion reduction with x-ray beam flux intensity. Additional spectra were measured from a freshly loaded $55 \mathrm{ppm}$ $\mathrm{Cu}$ in $\mathrm{CuCl}_{2}$ solution sample in the same cell as described above, under reduced flux of approximately $5 \times 10^{10}$ photons/s. The flux reduction was accomplished using three $0.1 \mathrm{~mm}$ aluminum foil sheets placed in the incident $\mathrm{x}$-ray beam. A substantial reduction in the rate of reduction of copper ions was observed, in the time-sequenced XAFS spectra measured from the sample. In addition to the brilliance of the incident beam, the yield of $e_{\mathrm{aq}}^{-}$to a larger extent is dependent on the $\mathrm{pH}$ conditions of the solution. ${ }^{21}$ Oxidation and/or reduction also depend on the type of cation in the solution. Lanthanum $L_{3}$-edge XAFS measurements made under similar conditions, on an aqueous solution of $1000 \mathrm{ppm}$ La powder with $2 \% \mathrm{HNO}_{3}$, show no such reduction effects. ${ }^{22}$ However, in addition to $\mathrm{Cu}^{2+}$ ions, attention may be required when dealing with aqueous solutions of metallic ions such as $\mathrm{Cr}^{3+}, \mathrm{Cu}^{+}, \mathrm{Fe}^{2+}, \mathrm{Fe}^{3+}$, and other ions that possess multiple oxidation states and, thus, have the possibility to undergo stepwise reduction and/or oxidation under favorable conditions.

\section{CONCLUSIONS}

In this study, we have observed the formation of copper particle clusters as a consequence of radiolysis of a dilute aqueous solution of $\mathrm{CuCl}_{2}$ by the incident x-ray radiation. The secondary radiation produced through scattering of the incident $\mathrm{x}$-rays is thought to cause the formation of hydrated electrons $\left(e_{\mathrm{aq}}^{-}\right)$, which in turn take part in the reduction of $\mathrm{Cu}^{2+}$ ions in the solution to their metallic state. Characterization of the clusters using XAFS yield results that are consistent with those observed in clusters grown in the solid state. The study shows a distribution of particle clusters ranging from $\sim 5$ to $\sim 10 \AA$ in diameter, beyond which the copper clusters show bulk-like characteristics. In the earliest stage of growth, the clusters exhibit significantly reduced $\mathrm{Cu}-\mathrm{Cu}$ distances and thermally-induced vibrational disorder, which are attributed to a somewhat greater degree of covalent bonding in the clusters in relation to metallic bonding in bulk copper. As a consequence, the absolute $\mathrm{Cu} K$ edge is shifted by +2.1 $\mathrm{eV}$ and the bulk modulus is estimated to be increased by $\sim 61 \%$ for the 19 -atom copper cluster in relation to their respective values for bulk ( $\mathrm{Cu}$ foil) copper. Fitting the firstshell features of the spectra reveals that the structure parameters are found to increase gradually and monotonically with time to attain values close to those of bulk copper.

\section{ACKNOWLEDGMENTS}

We wish to thank Steve Heald and other PNC-CAT beam line personnel and Robert Gordon of Simon Fraser University for their assistance during our experiments at the Advanced Photon Source. This research was funded by a Natural Sciences and Engineering Research Council of Canada (NSERC) research and equipment grant to A.J.A. and a grant from the Research Corporation to R.A.M. NSERC is also thanked for providing funds for this project through a Major Facility Access Grant. The U.S. Department of Energy, Office of Science, Basic Energy Sciences, under Contract No. W-31-109-Eng-38 (APS) and DE-FG03-97ER45628 (PNCCAT) supported the use of the Advanced Photon Source.

${ }^{1}$ C. D. Jonah, Radiat. Res. 144, 141 (1995).

${ }^{2}$ M. Freiberg, W. A. Mulac, K. H. Schmidt, and D. Meyerstein, J. Chem. Soc., Faraday Trans. 1 76, 1838 (1980).

${ }^{3}$ B. G. Ershov, E. Janata, M. Michaelis, and A. Henglein, J. Phys. Chem. 95, 8996 (1991).

${ }^{4}$ A. Henglein, J. Phys. Chem. B 104, 1206 (2000).

${ }^{5}$ G. Apai, J. F. Hamilton, J. Stohr, and A. Thompson, Phys. Rev. Lett. 43, 165 (1979).

${ }^{6}$ P. A. Montano, G. K. Shenoy, E. E. Alp, W. Schulze, and J. Urban, Phys. Rev. Lett. 56, 2076 (1986).

${ }^{7}$ M. A. Marcus, M. P. Andrews, J. Zegenhagen, A. S. Bommanavar, and P. Montano, Phys. Rev. B 42, 3312 (1990).

${ }^{8}$ A. Balerna, E. Bernieri, P. Picozzi, A. Reale, S. Santucci, E. Burrattini, and S. Mobilio, Phys. Rev. B 31, 5058 (1985).

${ }^{9}$ W. A. Bassett, A. J. Anderson, R. A. Mayanovic, and I. M. Chou, Z. Kristallogr. - New Cryst. Struct. 215, 711 (2000).

${ }^{10}$ M. Newville, P. Livins, Y. Yacoby, J. J. Rehr, and E. A. Stern, Phys. Rev. B 47, 14126 (1993)

${ }^{11}$ M. Newville, B. Ravel, D. Haskel, J. J. Rehr, E. A. Stern, and Y. Yacoby, Physica B 208\&209, 154 (1995).

${ }^{12}$ E. A. Stern, M. Newville, B. Ravel, Y. Yacoby, and D. Haskel, Physica B 208\&209, 117 (1995).

${ }^{13}$ A. L. Ankudinov, B. Ravel, J. J. Rehr, and S. D. Conradson, Phys. Rev. B 58, 7565 (1998). 
${ }^{14}$ E. Sevillano, H. Meuth, and J. J. Rehr, Phys. Rev. B 20, 4908 (1979).

${ }^{15}$ C.-Y. Cha, G. Ganteför, and W. Eberhardt, J. Chem. Phys. 99, 6308 (1993).

${ }^{16}$ R. E. Watson, M. L. Perlman, and J. F. Herbst, Phys. Rev. B 13, 2358 (1976).

${ }^{17}$ W. Ekardt, Phys. Rev. B 29, 1558 (1984).

${ }^{18}$ C. D. Gelatt, Jr., H. Ehrenreich, and R. E. Watson, Phys. Rev. B 15, 1613 (1977).
${ }^{19}$ Z. J. Zhang, J. Phys.: Condens. Matter 10, 495 (1998).

${ }^{20}$ O. L. Anderson, D. G. Isaak, and S. Yamamoto, J. Appl. Phys. 65, 1534 (1989).

${ }^{21}$ J. W. Boyle, J. A. Ghormley, C. J. Hochanadel, and J. F. Riley, J. Phys. Chem. 73, 2886 (1969).

${ }^{22}$ A. J. Anderson, S. Jayanetti, R. A. Mayanovic, W. A. Bassett, and I. M. Chou, Am. Mineral. (to be published). 